Chin. J. Astron. Astrophys. Vol.0 (200x) No.0, 000-000

(http://www.chjaa.org )

Chinese Journal of

Astronomy and

Astrophysics

\title{
Particle acceleration in astrophysical shear flows
}

\author{
Frank M. Rieger * and Peter Duffy \\ Department of Mathematical Physics, University College Dublin, Belfield, Dublin 4, Ireland
}

Received 2004; accepted 2004

\begin{abstract}
We consider the acceleration of particles due to a velocity shear in relativistic astrophysical flows. The basic physical picture and the formation of power law momentum spectra is discussed for a non-relativistic velocity field using a microscopic approach. We identify possible sites for shear acceleration in relativistic astrophysical jets and analyze their associated acceleration timescales. It is shown in particular that for a mean scattering time $\tau$ scaling with the gyro-radius, the acceleration timescale for gradual shear scales in the same manner as the synchrotron cooling timescale so that losses may no longer be able to stop the acceleration once it has started to work efficiently. Finally, the possible role of shear acceleration is discussed with reference to the relativistic jet in the quasar $3 \mathrm{C} 273$.
\end{abstract}

Key words: acceleration of particles — galaxies: active — galaxies: jets quasars: individual (3C273)

\section{INTRODUCTION}

Collimated relativistic outflows are observed in a variety of astrophysical environments, ranging from Active Galactic Nuclei (AGN), $\mu$-Quasars and neutron stars in binary systems to GammaRay Bursts (e.g., Zensus 1997; Mirabel \& Rodriguez 1999; Fender et al. 2004). There is growing evidence today that in all these sources the relativistic outflows, or at least those parts observed in the radio band, are essentially launched from an accretion disk (e.g., Livio 1999; Marscher et al. 2002; Fender et al. 2004). In several sources, monitoring of their small-scale radio structures has revealed outflowing components at (apparently) high superluminal velocities suggesting that the bulk plasma in these jets moves at relativistic speeds and small viewing angles towards the observer. Theoretical models based on the assumption of a simple, one-dimensional velocity structure have thus allowed valuable insights into the emission properties of such jets. In general however, such models are only adequate as a first approximation, as real jets are likely to be characterized by a significant shear in their flow velocity field. Using theoretical and observational arguments relevant for AGN jets for example, we show below that at least three different shear flow scenarios may be distinguished. In the presence of such shear flows, particle acceleration is likely to occur: In their pioneer work on the particle transport in non-relativistic

$\star$ E-mail: frank.rieger@ucd.ie 
gradual shear flows for example, Berezhko \& Krymskii (1981) (cf. also Berezhko 1981) showed that (steady-state) power law particle momentum spectra $f(p) \propto p^{-(3+\alpha)}$ may be formed if the scattering time follows a power law $\tau \propto p^{\alpha}, \alpha>0$. Later on, Earl, Jokipii \& Morfill (1988) rederived Parker's transport equation for non-relativistic gradual shear flows, but now augmented by new terms describing viscous (shear) and inertial effects. A relativistic generalization was achieved by Webb (1989) (cf. also Webb 1985 and Webb et al. 1994) based on a mixed-frame approach, where the scattering operator is evaluated in the comoving frame, and applied to the cosmic ray transport in our galaxy. Particle acceleration in non-gradual relativistic shear flow has been considered by Ostrowski (1990, 1998, 2000; cf also Stawarz \& Ostrowski 2002) based on Monte Carlo simulations, showing that very flat particle momentum spectra may be possible. More recently, Rieger \& Mannheim (2002) have studied the acceleration of particles in rotating and shearing flows with application to relativistic AGN jets. Based on the insights gained from these works, shear acceleration in astrophysical jets appears in some respects unavoidable. Whether shear-accelerated particles may significantly contribute to the emission in a given energy band seems thus not a matter of occurrence but simply a matter of efficiency of the underlying shear acceleration mechanism.

\section{A SIMPLIFIED APPROACH TO SHEAR ACCELERATION}

Shear acceleration is based on the idea that energetic particles may gain energy by scattering off systematically moving small-scale magnetic field irregularities. These scattering centres (SCs) are thought to be embedded in a collisionless (gradual) shear flow such that the velocity of a certain SC corresponds to the local flow velocity, i.e. in contrast to second order Fermi acceleration a random motion of SCs is neglected. Hence the acceleration process essentially draws on the kinetic energy of the background flow. Scattering of particles is assumed to occur in such a way that the particle momentum is randomized in direction and its magnitude is conserved in the local comoving fluid/scattering frame. Obviously, if there is no velocity shear (or rotation) present and the flow is not diverging, particles will neither gain energy nor momentum merely due to scattering. However, in the presence of a velocity shear in the flow, the momentum of a particle travelling across the shear changes, so that a net increase may occur. The physical argument perhaps becomes best transparent in the microscopic picture for a non-relativistic continuous shear flow (cf., Jokipii \& Morfill 1990) with velocity profile given by $\boldsymbol{u}=u_{z}(x) \boldsymbol{e}_{z}$. Consider a particle with velocity vector $\boldsymbol{v}=\left(v_{x}, v_{y}, v_{z}\right)$, relativistic mass $m$ and initial momentum $\boldsymbol{p}_{1}$ relative to local flow frame. Within a scattering time $\tau$ (assumed to be independent of momentum) the particle will travel a distance $\delta x=v_{x} \tau$ across the shear, so that for a gradual shear flow the flow velocity will have changed by an amount $\delta \boldsymbol{u}=\delta u \boldsymbol{e}_{\boldsymbol{z}}$, where $\delta u=\left(\partial u_{z} / \partial x\right) \delta x$. Contenting ourselves with a Galilean transformation for the nonrelativistic flow speeds involved, the particle's momentum relative to the flow will have changed to $\boldsymbol{p}_{2}=\boldsymbol{p}_{1}+m \delta \boldsymbol{u}$, i.e.

$$
p_{2}^{2}=p_{1}^{2}+2 m \delta u p_{1, z}+m^{2}(\delta u)^{2} .
$$

As the next scattering event preserves the magnitude of the particle momentum relative to the local flow speed, the particle magnitude will have this value in the local flow frame and hence a net increase in momentum may occur with time. By using spherical coordinates, defining $<\Delta p / \Delta t>=2<\left(p_{2}-p_{1}\right)>/ \tau$ as the average rate of momentum change and $<\Delta p^{2} / \Delta t>=$ $2<\left(p_{2}-p_{1}\right)^{2}>/ \tau$ as the average rate of momentum dispersion, it can be shown that averaging over solid angles assuming a nearly isotropic particle distribution results in (cf., Jokipii \& Morfill 1990)

$$
\left\langle\frac{\Delta p^{2}}{\Delta t}\right\rangle=\frac{2}{15} p^{2}\left(\frac{\partial u_{z}}{\partial x}\right)^{2} \tau
$$




$$
\left\langle\frac{\Delta p}{\Delta t}\right\rangle=\frac{4}{15} p\left(\frac{\partial u_{z}}{\partial x}\right)^{2} \tau
$$

both being related by

$$
\left\langle\frac{\Delta p}{\Delta t}\right\rangle=\frac{1}{2 p^{2}} \frac{\partial}{\partial p}\left(p^{2}\left\langle\frac{\Delta p^{2}}{\Delta t}\right\rangle\right) .
$$

Note that a momentum-dependent scattering time obeying a power law of the form $\tau \propto p^{\alpha}$ may be accommodated by replacing $4 \rightarrow(4+\alpha)$ in Eq. (3). We may then gain further insights into possible particle momentum spectra under viscous shear energy changes by studying the (simplified) steady state transport equation for the phase space distribution function $f(p)$

$$
\frac{1}{p^{2}} \frac{\partial}{\partial p}\left(p^{2}\left\langle\frac{\Delta p}{\Delta t}\right\rangle f(p)\right)-\frac{1}{2 p^{2}} \frac{\partial^{2}}{\partial p^{2}}\left(p^{2}\left\langle\frac{\Delta p^{2}}{\Delta t}\right\rangle f(p)\right)=Q \delta\left(p-p_{0}\right),
$$

assuming particles to be injected with momentum $p_{0}$ and $\alpha>0$. Solving Eq. (15) one immediately obtains

$$
f(p) \propto p^{-(3+\alpha)} H\left(p-p_{0}\right),
$$

where $H(p)$ is the Heaviside step function. Note that for a mean scattering time scaling with the gyro-radius (Bohm case), i.e. $\tau \propto p, \alpha=1$, this gives $f(p) \propto p^{-4}$ and thus a power law particle number density $n(p) \propto p^{-2}$ translating into a synchrotron emissivity $j_{\nu} \propto \nu^{-1 / 2}$. For a Kolmogorov-type $(\alpha=1 / 3)$ or Kraichnan-type $(\alpha=1 / 2)$ scaling on the other hand, much flatter spectra are obtained.

\section{POSSIBLE VELOCITY SHEAR SITES IN ASTROPHYSICAL JETS}

In general, at least three different shear scenarios may be distinguished (cf. Rieger \& Duffy 2004):

\subsection{Gradual velocity shear parallel to the jet axis}

Observationally, we have strong direct evidence for internal jet stratification (e.g. such as a fast moving inner spine and a slower moving boundary layer) on the pc-scale in sources such as 3C353, M87, Mkn 501 or PKS 1055+018 (e.g., Swain et al. 1998; Attridge et al. 1999; Perlman et al. 1999; Edwards et al. 2000). Moreover, there is a multitude of indirect, phenomenological evidence for internal jet stratification including the requirements for the unification of BL Lacs and FR I (e.g., Chiaberge et al. 2000) or results from hydrodynamical jet simulations (e.g., Aloy et al. 2000; Gomez 2002). In particular, Laing et al. (1999) have argued recently that the intensity and polarization systematics in kpc-scale FR I jets are suggestive of a radially

(continuously) decreasing velocity profile $v_{z}(r)$.

\subsection{Non-gradual velocity shear parallel to the jet axis}

In general, a gradual shear analysis may be justified if the flow velocity changes continuously and the particle mean free path $\lambda$ is much smaller than the width of the shear region. However, when a particle becomes so energetic that its mean free path becomes larger than the width of the velocity transition layer, it will essentially experience a non-gradual shear flow discontinuity. Ostrowski $(1990,1998)$ for example, has convincingly argued that the jet side boundary between the relativistic jet interior and its ambient medium may represent a natural realization of such a non-gradual (discontinuous) relativistic velocity shear. 


\subsection{Gradual velocity shear across the jet}

Astrophysical jets are also likely to exhibit a significant velocity shear perpendicular to their jet axis ('transversal shear'). In particular, several independent arguments suggest that astrophysical jets may be characterized by an additional rotational velocity component: The strong correlation between the disk luminosity and the bulk kinetic power in the jet (e.g., Rawlings \& Saunders 1991), the successful applications of jet-disk symbiosis models (e.g., Falcke \& Biermann 1995) and the observational evidence for a disk-origin of jets (e.g., Marscher et al. 2002) or periodic variability (e.g., Camenzind \& Krockenberger 1992) suggest that a significant amount of accretion energy and hence rotational energy of the disk is channeled into the jet (due to angular momentum conservation). Moreover, internal jet rotation is generally implied in theoretical MHD models of jets as magnetized disk winds (e.g., Vlahakis \& Tsinganos 1998; Sauty et al. 2002). Finally, direct observational support for internal jet rotation has been recently established for stellar jets (e.g., Coffey et al. 2004). Based on such arguments, Rieger \& Mannheim (2002) have analyzed the acceleration of particles in rotating and shearing, relativistic AGN jets. Their analysis confirmed the results of Berezhko \& Krymskii (1981) for the case of non-relativistic Keplerian rotation, but also showed that inclusion of centrifugal effects may lead to a flattening of the spectra.

\section{ON THE EFFICIENCY OF SHEAR ACCELERATION}

Rieger \& Duffy (2004) have recently studied the acceleration potential for the different shear scenarios distinguished above by estimating their associated acceleration timescales. For a gradual shear flow parallel to the jet axis (case 3.1.) with a velocity profile decreasing linearly from relativistic to non-relativistic speeds over a scale $\Delta r$, they found a minimum acceleration timescale

$$
t_{\text {grad }, \|} \sim \frac{3}{\lambda} \frac{(\Delta r)^{2}}{c \gamma_{b}(r)^{4}}
$$

where $\gamma_{b}(r)>1$ is the (position-dependent) bulk Lorentz factor of the flow and $\lambda$ the particle mean free path. By comparing the acceleration with the synchrotron cooling timescale for parameters appropriate for pc-scale radio jets, it can be shown that efficient electron acceleration is quite restricted and only possible if $\Delta r$ is very small, while proton acceleration is much more favourable since $\lambda_{\text {proton }} \gg \lambda_{\text {electron}}$. Perhaps most interestingly however, for a particle mean free path scaling with the gyro-radius, i.e. $\lambda \propto \gamma$, both the acceleration and the cooling timescale have the same dependency on the particle Lorentz factor $\gamma$. In such a case losses are no longer able to stop the acceleration process once it has started to work efficiently. Particle escape, when the $\lambda$ becomes larger than the width of the jet, or cross-field diffusion however, may then still represent important constraints on the maximum attainable energy.

For a non-gradual, relativistic velocity jump (case 3.2.) on the other hand, the minimum acceleration timescale is of order (cf. Ostrowski 1990, 1998)

$$
t_{\mathrm{acc}} \sim 10 \frac{r_{g}}{c} \quad \text { provided } \quad r_{g}>\Delta r
$$

where $r_{g}$ denotes the gyro-radius of the particle and $\Delta r$ the width of the transition layer, so that the acceleration timescale in this case is proportional to $\gamma$. Due to the condition $r_{g}>$ $\Delta r$, efficient acceleration of electrons is unlikely given their associated rapid radiation losses. However, the acceleration of protons may be well possible until their particle mean free path becomes larger than the width of the jet.

Finally, for a shear flow with relativistic $v_{z}$ and azimuthal Keplerian rotation profile $\Omega(r) \propto$ 
$\left(r_{\text {in }} / r\right)^{3 / 2}$ for example (case 3.3.), the acceleration timescale obeys

$$
t_{\mathrm{grad}, \perp} \propto \frac{1}{\lambda}\left(\frac{r}{r_{\mathrm{in}}}\right)^{3}
$$

assuming the flow to be radially confined to $r_{\text {in }} \leq r \leq r_{j}$ where $r_{j}$ is the jet radius. In general, efficient particle acceleration requires a region with significant rotation. For such a case the higher energy emission will naturally be concentrated closer to the axis (i.e. towards smaller radii). Again however, electron acceleration is usually very restricted (i.e. only possible for $r \sim r_{\text {in }}$ ), while proton acceleration is much more favourable (i.e. possible up to $r \sim r_{j}$ ). If $\lambda$ scales with the gyro-radius, the acceleration and the cooling timescale will again have the same dependency on $\gamma$ and thus losses will not longer be able to stop the acceleration process once it has started to work efficiently.

\section{POSSIBLE APPLICATIONS AND RELEVANCE}

Observational and theoretical evidence suggest that astrophysical jets are characterized by a significant velocity shear. While internal jet rotation for example, is likely to be present at least in the initial parts of the jet, a significant longitudinal velocity shear (parallel to the jet axis) prevailing all along the jet might be expected for most sources. In general, the shear acceleration mechanism analyzed above may thus operate over many length scales. Thus, in contrast to shock acceleration, shear acceleration is usually not constrained to localized regions in the jets. Besides the possibility that shear acceleration may account for a second population of energetic particles in the jet (e.g. in addition to shock-accelerated ones), shear acceleration might be of particular relevance for our understanding of the continuous radio and optical emission observed from several sources (e.g., Meisenheimer et al. 1997; Scarpa et al. 1999; Jester et al. 2001). In the case of the quasar 3C $273(z=0.158)$ for example, the optical spectral index is found to vary only very smoothly along the (large-scale) jet with no signs of strong synchrotron cooling at any location in the jet, e.g. between knots, contrary to expectations from shock acceleration scenarios (Jester et al. 2001), suggesting the need of a continuous re-acceleration mechanism (cf. also Meisenheimer et al. 1997). The jet in 3C 273 appears to be highly relativistic even on kpc-scales with a typical Doppler factor $D \sim 5$ (cf. Sambruna et al 2001) so that longitudinal shear acceleration of particles may probably work efficiently nearly all along the jet. Moreover, there is also evidence for helical bulk motion in the large-scale jet (e.g., Bahcall et al. 1995) and internal jet helicity (e.g., oscillating ridge line, double helical pattern, periodicity) on the VLBI mas-scale and below (e.g., Krichbaum et al. 2000, Camenzind \& Krockenberger 1992), suggesting that particle acceleration due to internal jet rotation (cf. Rieger \& Mannheim 2002) may contribute on pc-scale and perhaps even on larger scales.

Acknowledgements FMR acknowledges support by a Marie-Curie Individual Fellowship (MCIF2002-00842). Discussions with V. Bosch-Ramon and K. Mannheim are gratefully acknowledged.

\section{References}

Aloy M.-A., Gomez J.-L., Ibanez J.-M. et al., 2000, ApJ, 520, L85

Attridge J. M., Roberts D. H., Wardle J. F. C., 1999, ApJ, 518, L87

Bahcall J. N., Kirhakos S., Schneider D. P. et al., 1995, ApJ, 452 L91

Berezhko E. G., 1981, JETP Letters 33, 399

Berezhko E. G., Krymskii G. F., 1981, Sov. Astr. Lett., 7, 352

Camenzind M., Krockenberger M., 1992, A\&A, 255, 59 
Chiaberge M., Celotti A., Capetti A., Ghisellini G., 2000, A\&A, 358, 104

Coffey D., Bacciotti F., Woitas J. et al., 2004, ApJ, 604, 758

Earl J. A., Jokipii J. R., Morfill G., 1988, ApJ, 331, L91

Edwards P. G., Giovannini G., Cotton W. D. et al., 2000, PASJ, 52, 1015

Falcke H., Biermann P.L., 1995, A\&A, 293, 665

Fender R., Wu. K., Johnston H. et al., 2004, Nature, 427, 222

Gomez J.-L., 2002, In: A. W. Guthmann et al., eds., Relativistic Flow in Astrophysics (LNP 589),

Berlin: Springer, 169

Jester S., Röser H-J., Meisenheimer K. et al., 2001, A\&A, 373, 447

Jokipii J. R., Morfill G. E., 1990, ApJ, 356, 255

Krichbaum T., Witzel A., Zensus, J. A., 2000, In: J. E. Conway et al., eds., Proceedings of the 5th European VLBI Network Symposium (Onsala), p. 25.

Laing R. A., Parma P., de Ruiter H. R, Fanti R., 1999, MNRAS, 306, 513

Livio M., 1999, Phys. Rep., 311, 225

Marscher, A.P., Jorstad, S.G., Gomez, J.-L. et al., 2002, Nature, 417, 625

Meisenheimer K., Yates M. G., Röser H-J, 1997, A\&A, 325, 57

Mirabel I. F., Rodriguez L. F., 1999, ARA\&A, 37, 409

Ostrowski M., 1990, A\&A, 238, 435

Ostrowski M., 1998, A\&A, 335, 134

Ostrowski M., 2000, MNRAS, 312, 579

Perlman E. S., Biretta J. A., Zhou F. et al., 1999, AJ, 117, 2185

Rawlings S., Saunders R., 1991, Nature, 349, 138

Rieger F. M., Mannheim K., 2002, A\&A, 396, 833

Rieger F. M., Duffy P., 2004, ApJ, 617, 155

Sambruna R. M., Urry C. M., Tavecchio F. et al., 2001, ApJ, 549, L161

Scarpa R., Urry C. M., Falomo R., Treves A., 1999, ApJ, 526, 643

Stawarz, E., Ostrowski, M., 2002, ApJ, 578, 763

Swain M. R., Bridle A. H., Baum S. A., 1998, ApJ, 507, L29

Sauty C., Tsinganos K., Trussoni E., 2002, In: A. W. Guthmann et al., eds., Relativistic Flow in Astrophysics (LNP 589), Berlin: Springer, 41

Vlahakis N., Tsinganos K., 1998, MNRAS, 298, 777

Webb G. M., 1985, ApJ, 296, 319

Webb G. M., 1989, ApJ, 340, 1112

Webb G. M., Jokipii J. R., Morfill G. E., 1994, ApJ, 424, 158

Zensus J. A., 1997, ARA\&A, 35, 607

This paper was prepared with the ChJAA LATEX macro v1.0. 